\title{
High-field impurity magneto-optics of Si:Se
}

\author{
K. L. Litvinenko, ${ }^{1, *}$ M. Pang, ${ }^{1}$ Juerong Li,${ }^{1}$ E. Bowyer, ${ }^{1}$ H. Engelkamp, ${ }^{2}$ V. B. Shuman, ${ }^{3}$ L. M. Portsel,,${ }^{3}$ A. N. Lodygin, ${ }^{3}$ \\ Yu. A. Astrov, ${ }^{3}$ S. G. Pavlov, ${ }^{4}$ H.-W. Hübers, ${ }^{4,5}$ C. R. Pidgeon, ${ }^{6}$ and B. N. Murdin ${ }^{1}$ \\ ${ }^{1}$ Advanced Technology Institute and SEPNet, University of Surrey, Guildford GU2 7XH, United Kingdom \\ ${ }^{2}$ High Field Magnet Laboratory, Institute for Molecules and Materials, Radboud University Nijmegen, Toernooiveld 7, NL-6525 ED \\ Nijmegen, The Netherlands \\ ${ }^{3}$ Ioffe Physical-Technical Institute, Politekhnicheskaya 26, 194021 St. Petersburg, Russia \\ ${ }^{4}$ Institute of Planetary Research, German Aerospace Center (DLR), Rutherfordstraße 2, 12489 Berlin, Germany \\ ${ }^{5}$ Technische Universität Berlin, Institut für Optik und Atomare Physik, Hardenbergstrasse 36, 10623 Berlin, Germany \\ ${ }^{6}$ Institute of Photonics and Quantum Sciences, SUPA, Heriot-Watt University, Edinburgh EH14 4AS, United Kingdom
}

(Received 30 June 2014; revised manuscript received 18 August 2014; published 8 September 2014)

\begin{abstract}
Just as phosphorus in silicon produces a hydrogenic defect, the double donor selenium in silicon is an analog of helium. We have measured the impurity absorption spectrum at high magnetic field, and we show that the odd-parity excited states of Si:Se behave identically to those of Si:P. This fact allows us to isolate the electron-electron interactions (exchange and correlation) in the ground state from the quadratic Zeeman effect. The field tuning allows us to put upper limits on the strength of some of these interactions (e.g., at $30 \mathrm{~T}$ the electron-electron correlation interaction in the ground state of $\mathrm{Se}$ is less than about $40 \mu \mathrm{eV}$; at $30 \mathrm{~T}$ the quadratic Zeeman energy in the ground state of $\mathrm{P}$ is less than about $200 \mu \mathrm{eV}$ ).
\end{abstract}

DOI: 10.1103/PhysRevB.90.115204

PACS number(s): 03.67.Lx, 71.55.-i, 32.80.Rm, 78.20.Ls

\section{INTRODUCTION}

Group-V donors in silicon are hydrogenic analogs with a single extra electron that is not used in the bonding, orbiting around the singly positively charged ion. The spectrum can be easily found from an effective mass model, i.e., by simply scaling the hydrogen calculation with the mass and dielectric constant (see Table I), so long as the anisotropy of the electron effective mass is taken into account. This model successfully predicts the energy spectrum of the excited states of the shallow single donors, and their magnetic field dependence. The scaling means that experiments on Si:P at $30 \mathrm{~T}$ have been shown to correspond to hydrogen at $10^{5} \mathrm{~T}$, the highest field observed on a white dwarf [1,2]. This model also predicts the wave-function radii, for example, that of the $2 p_{0}$ state of $\mathrm{Si}: \mathrm{P}$ is $5.4 \mathrm{~nm}$.

The ground state is not well described by the effective mass model because of the breakdown of the envelope function approximation for tightly bound orbits. From the scaled Bohr model the radius of state $n$ is $r_{n}=a_{0} E_{h} / 2 \varepsilon_{r} E_{n}$, where $\varepsilon_{r}$ is the relative dielectric constant and $E_{n}$ is its binding energy, so the radius of the ground state with binding energy $45.59 \mathrm{meV}$ (at $4 \mathrm{~K}$ ) of Si:P is $1.4 \mathrm{~nm}$, which is only a few times larger than the lattice constant, $0.54 \mathrm{~nm}$ (at $4 \mathrm{~K}$ ) [3]. The excited states do not suffer this way because of the much larger extent and the small probability density at the center. The even parity excited states (e.g., $2 s$, etc.) have slightly larger penetration to the core and small extra shifts, and splittings and changes to the matrix elements are apparent. The ground state of impurities in silicon and the field dependence is needed for certain quantum information schemes where electron and nuclear spins are used [4], and the excited states are also important for some gating control architectures [5]. In interpretation of the electric

*Corresponding author: k.litvinenko@ surrey.ac.uk dipole (Rydberg) spectrum, one usually treats the ground-state energy as a free parameter [1], and the calculated transitions are in excellent agreement with the theory.

Helium at high field is of great interest for the study of magnetic white dwarfs [6] because it provides an independent control on the assignment of the field from hydrogen spectra, which are very complex [1]. Of course, there are no laboratory experiments up to fields of 1 atomic unit (a.u.), but unlike hydrogen there are only very few calculations of the Rydberg excitation spectrum of free helium atoms because of the difficulty of incorporating the correlation and exchange energies. For example, there are no theoretical reports of the excited states with magnetic quantum number $M=+1$ (presumably because in astrophysical situations where the field is inhomogeneous, states that tune rapidly with field are less interesting). The electron-electron effects make the calculations hard but they produce an interesting phase transition at very high field; the ground state of helium at low field is a singlet state $1^{1} 0^{+}$(with spins antiparallel, known as parahelium, see Table II), but at $B \sim 0.76 B_{0}$ the lowest triplet state $1^{3}(-1)^{+}$(orthohelium, spins parallel) drops below it [7]. The resulting dipole can produce a new type of bond that is magnetic in origin rather than electric [8].

The group-VI chalcogen atoms are well-known deep double donors when on an isolated substitutional silicon site. They are interesting for quantum information applications because they can be addressed with table-top lasers in the midinfrared. The advantages of silicon over other hosts are the same as those outlined in previous studies of Si:P [1]. The zero-field absorption spectrum is analogous to that of free helium atoms [9], and in this work we investigate experimentally the high-field spectrum of a range of excitedstate transitions in neutral selenium centers and compare them with the predictions for free helium, up to fields equivalent to $100,000 \mathrm{~T}$. 
TABLE I. Atomic units used, where $\kappa=4 \pi \varepsilon_{0}$, and scaled atomic units for silicon where $\kappa \rightarrow \varepsilon_{r} \kappa$ and $m_{e} \rightarrow m_{t}$, the transverse effective mass [1]. Note that mass anisotropy in silicon (longitudinal mass is larger) means that all silicon states are slightly deeper and smaller than implied by simple scaling.

\begin{tabular}{lccc}
\hline \hline Atomic unit & & Free hydrogen & Silicon \\
\hline Energy (hartree) & $E_{h}=m_{e} e^{4} / \kappa^{2} \hbar^{2}$ & $27.2 \mathrm{eV}$ & $0.0399 \mathrm{eV}$ \\
Magnetic field & $B_{0}=m_{e}^{2} e^{3} / \kappa^{2} \hbar^{3}$ & $235,000 \mathrm{~T}$ & $65.6 \mathrm{~T}$ \\
Length (bohr) & $a_{0}=\kappa \hbar^{2} / m_{e} e^{2}$ & $0.056 \mathrm{~nm}$ & $3.17 \mathrm{~nm}$ \\
\hline \hline
\end{tabular}

\section{THEORY}

The high-field spectrum of hydrogen is difficult to calculate in the situation where the Coulombic binding of the electron with the nucleus is comparable with the cyclotron energy due to the magnetic field; in very low field the symmetry of the potential is essentially spherical with a small cylindrical perturbation, and in very high field it is the reverse. At intermediate field (i.e., near 1 atomic unit of magnetic field, Table I) the symmetry of the potential is no longer well defined. In atomic units, the Hamiltonian for a gauge appropriate for a basis of hydrogenic spherical harmonics is

$$
H=H_{0}+\mathbf{B} \cdot \mathbf{L}+\frac{1}{8}(\mathbf{B} \times \mathbf{r})^{2},
$$

where the first term on the right is the Coulombic energy responsible for the Rydberg series, the second is the Zeeman energy, and the third is the quadratic Zeeman energy. The latter results in a strong mixing of basis states and significant computational effort is required. The Hamiltonian of Eq. (1) does not include spin; if required, we simply add $\pm B / 2$ to the result (taking $g=2$ ). Ionization energies may be found by noting that the continuum edge energy is $B / 2 \pm B / 2$.

In silicon the continuum is replaced by the conduction band, which, reflecting the cubic lattice, has six minima displaced along each of the principal axes in momentum space. The states with zero group velocity thus have nonzero phase velocity, which is simply an effect of the periodic medium. For a magnetic field along a crystal axis, two minima are longitudinal and four are transverse, and mass anisotropy means these have different field dependences, e.g., there are two kinds of $2 p_{-}$ levels - those with the field along $z$ and those with field along $x$ (we refer to the latter as $2 p_{-x}$ ), etc. Two methods for taking into account the anisotropy in the mass are described in Refs. [1] and [2].
In comparison with hydrogen, the level spectrum of helium and its magnetic field dependence is even more difficult to calculate due to the interaction between the two electrons, called the electron-electron (Coulomb) correlation, as well as the possibility of spin-spin (exchange) interactions. The triplet ground state $1^{3}(-1)^{+}$demonstrates an increase of exchange energy by approximately 2 orders of magnitude when the field is changed from 0 to $100 B_{0}$, because of the constriction of the wave function by the field [7]. The spin singlet states, such as the ground state $1^{1} 0^{+}$, do not produce exchange energy changes with field. However, the correlation energy of the $1^{1} 0^{+}$state monotonically increases by a factor of 2 with the field strength up to $100 B_{0}$ [7]. In what follows the ground state is always the singlet, and we investigate only electric dipole transitions that conserve spin. We therefore use the common simplified notation from the semiconductor literature, e.g., $2 p_{+}$refers to $2{ }^{1} P_{+1}$ or $1^{1}(+1)^{+}[10]$.

\section{EXPERIMENT}

The growth and characterization of the sample under investigation ("72-8a") is fully described in [11]. It was a 660$\mu \mathrm{m}$-thick wafer from float-zone-grown $\langle 100\rangle$ monocrystalline natural silicon. A method based on diffusion from the gas phase was used in order to produce a sample with Se doping of 2.6 $\times 10^{15} \mathrm{~cm}^{-3}$. The sample was mechanically and chemically polished to a $0.5^{\circ}$ wedge and mounted in helium exchange gas at $\mathrm{T}=1.4 \mathrm{~K}$ in the bore of a 33- $\mathrm{T}$ water-cooled Bitter magnet. Midinfrared radiation from a Fourier transform interferometer (Bruker IFS-113v) was brought to the sample by an evacuated beam line and a focusing cone. An optical window fitted at the bottom of the magnet allowed us to collect the transmitted radiation by means of an external liquid-nitrogen-cooled mercury cadmium telluride detector. The light propagation was parallel to the magnetic field direction and perpendicular to the sample surface (Faraday geometry).

\section{RESULTS}

Chalcogen dopants give rise to a number of different donor centers in silicon $[12,13]$. These centers can involve one $\left(\mathrm{Se}^{0}\right)$, two $\left(\mathrm{Se}_{2}^{0}\right)$, or more atoms and can be either neutral or ionized. Sections of the zero-field spectrum are shown in Fig. 1, corresponding to the transitions of neutral $\mathrm{Se}$ and $\mathrm{Se}_{2}$ centers. Also shown in Fig. 1 is the spectrum of Si:P from Ref. [1]

TABLE II. Lowest energy states of helium from Ref. [10]. Energies are in atomic units and are relative to the double-ionization threshold. The constants of the motion when $B \neq 0$ are total spin $S, z$ component of spin $S_{z}$, spatial parity, and magnetic quantum number $M$, leading to a notation $v_{S_{z}}^{2 S+1} M^{ \pm}$, where $v=1,2,3, \ldots$ labels the excitation state within the subspace defined by the constants of motion. N.B.: One electron must remain in the ground state; exciting both spins requires more than $2.9 E_{h}$.

\begin{tabular}{|c|c|c|c|c|c|}
\hline \multicolumn{3}{|c|}{ Singlet parahelium } & \multicolumn{3}{|c|}{ Triplet orthohelium } \\
\hline \multicolumn{2}{|c|}{$B=0$} & \multirow{2}{*}{$\frac{B \neq 0}{v_{S_{z}}^{2 S+1} M^{ \pm}}$} & \multicolumn{2}{|c|}{$B=0$} & \multirow{2}{*}{$\frac{B \neq 0}{v_{S_{z}}^{2 S+1} M^{ \pm}}$} \\
\hline Energy $/ E_{h}$ & $n^{2 S+1} L_{M}$ & & Energy $/ E_{h}$ & $n^{2 S+1} L_{M}$ & \\
\hline-2.903724377 & $1^{1} S_{0}$ & $1^{1} 0^{+}$ & & & \\
\hline-2.145974046 & $2^{1} S_{0}$ & $2^{1} 0^{+}$ & -2.175229378 & $1^{3} S_{0}$ & $1^{3} 0^{+}$ \\
\hline \multirow[t]{2}{*}{-2.1238430864} & $2^{1} P_{0}$ & $1^{1} 0^{-}$ & -2.133164191 & $1^{3} P_{0}$ & $1^{3} 0^{-}$ \\
\hline & $2^{1} P_{ \pm 1}$ & $1^{1}( \pm 1)^{+}$ & & $1^{3} P_{ \pm 1}$ & $1^{3}( \pm 1)^{+}$ \\
\hline
\end{tabular}




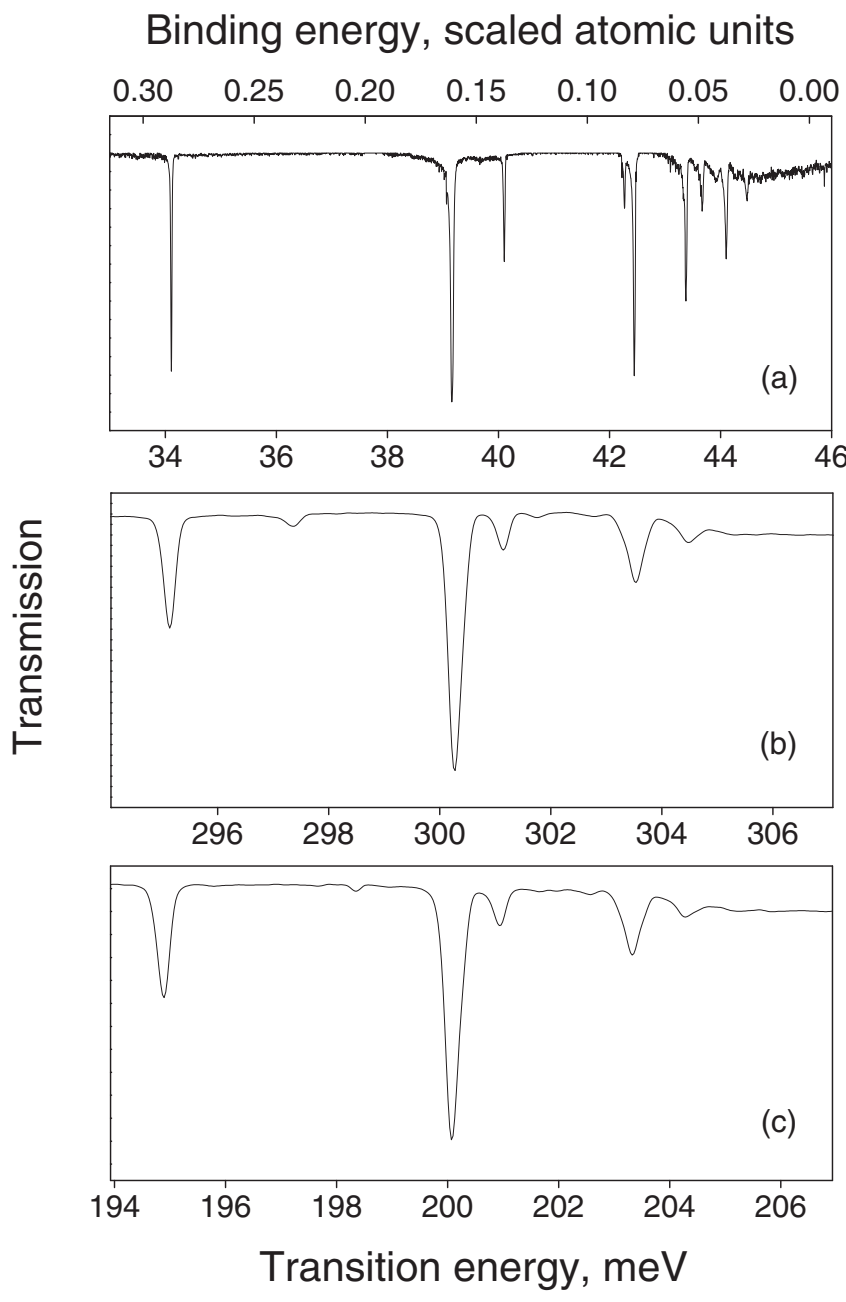

FIG. 1. Zero-field transmission spectrum of Si:P (a), Si:Se (b), and $\mathrm{Si}: \mathrm{Se}_{2}$ (c) centers showing the strong similarity between them.

for comparison. In each panel of the figure the horizontal scale span is identical and the continuum edges have been lined up. The similarity between the spectra is striking. The field dependence of Si:P and Si:Se is shown in Fig. 2(a). The correspondence between the observed transitions in $\mathrm{P}$ and $\mathrm{Se}$ is clear. The symmetry breaking due to the mass anisotropy results in the appearance of additional Se and $\mathrm{P}$ transitions not present for $\mathrm{H}$ or $\mathrm{He}$, such as the $2 p_{0 x}$ and $3 p_{0 x}$. Note that the character of $3 p_{0 x}$ and $2 p_{ \pm x}$ swaps at $0.2 B_{0}$ and that Se has an extra transition at $0.0025 E_{h}$ that is weakly allowed at low field by the broken symmetry due to the central cell.

\section{DISCUSSION}

\section{A. The excited states}

As already mentioned, the Si:P odd-parity excited states are very well described by a hydrogenic model with an anisotropic effective mass but without any central cell correction or valley mixing. The correspondence between the excited states of $\mathrm{Si}: \mathrm{P}$ and $\mathrm{Si}: \mathrm{Se}$ at zero field has been noted before [9], but now we are able to compare the magneto-optics of $\mathrm{Si}: \mathrm{Se}$ with the calculations for the magneto-optics of free helium. We examine first the correspondence of the excited states of
Si:Se with orthohelium using the spectra shown in Figs. 1 and 2. For example, the transition to a Se $2 p_{-}$excited state corresponds well with the transition to the $1^{1}(-1)^{+}$state of $\mathrm{He}$, shown in Fig. 2(b). It is clear from Fig. 2(c) that the excited states of helium very closely resemble those of hydrogen, because only one of the two electrons is excited, leaving behind the remaining $\mathrm{e}^{-}$, which is very tightly bound to the $\mathrm{He}^{2+}$ nucleus. The excited electron effectively sees a pointlike singly positively charged ion, especially in odd-parity states. The effects of exchange and correlation, i.e., the deviations from hydrogen, decrease further with increasing quantum number. The excited states of Si:Se are similarly expected to be well described by the Si:P hydrogenic model. Using the same scaling rules above, the $\mathrm{Se}^{+}$ion, which has binding energy of $593 \mathrm{meV}$ [14], has a radius of $0.1 \mathrm{~nm}$, far smaller than the radius of the hydrogenic $2 p_{0}$ state mentioned above (and even smaller than the lattice constant of silicon crystal). In silicon, the extra exchange and correlation effects are greatly reduced in comparison with free orthohelium, because the distance between the excited electron and the unexcited electron is so large.

From the experiment it is possible to compare splittings in the excited states without influence from the ground state by looking at the difference between transitions. For example, the $2 p_{+}$to $2 p_{-}$splitting is clearly identical in Se and in $\mathrm{P}$, as shown in Fig. 3(a) (diamonds). This is just as expected - the exchange and correlation energies for the Se $2 p_{+}$and $2 p_{-}$should be the same because they have the same spatial probability density, so the difference should remove any such corrections, producing the same excited-state splitting energies as for P. Unfortunately, there are few calculations for helium energy levels, and we have not found any for the $2 p_{+}\left[1^{1}(+1)^{+}\right]$. Figure 3(b) shows the comparison between the $2 p_{0}$ to $2 p_{-}$splitting using data taken from Refs. [15] and [16], with a clear difference between $\mathrm{He}$ and $\mathrm{H}$ due to the different probability densities and hence different exchange and correlation energies for the $\mathrm{He} 2 p_{0}$ to $2 p_{-}$states. Experimentally in silicon, transitions involving these two cannot both be observed for the same (longitudinal) valley with the field geometry used (Faraday configuration), but for the transverse valleys the $2 p_{ \pm x}$ and $2 p_{0 x}$ also show a negligible difference between Se and $\mathrm{P}$ [down triangles in Fig. 3(a)]. We conclude that the excited states of Se and P are identical, because they are very far from the core, and that the unexcited electron in the Se has a negligible influence on the energy, unlike free helium atoms, which have small electron-electron effects in the excited states.

$\mathrm{Si}: \mathrm{Se}_{2}$ also has a very similar excited-state spectrum (Fig. 1), and analysis of the high magnetic field dependence in the same way shows that it, too, has excited states that are identical to Si:P (data not shown) for exactly the same reasons, namely, that the $\mathrm{Se}_{2}^{+}$ion is extremely compact, and the three electrons that are not used in the bonding, which remain unexcited, have no influence on the energy of the excited electron.

We note in passing that we have not investigated any triplet excited states (the electric-dipole excitations from the singlet ground state leave the spin of the excited electron unchanged), and we have not reached the field at which the Se $2 p_{-}$triplet state drops below the $1 s$ singlet. The helium crossover occurs at $0.76 B_{0}$, which would be at around $50 \mathrm{~T}$ in silicon and is 

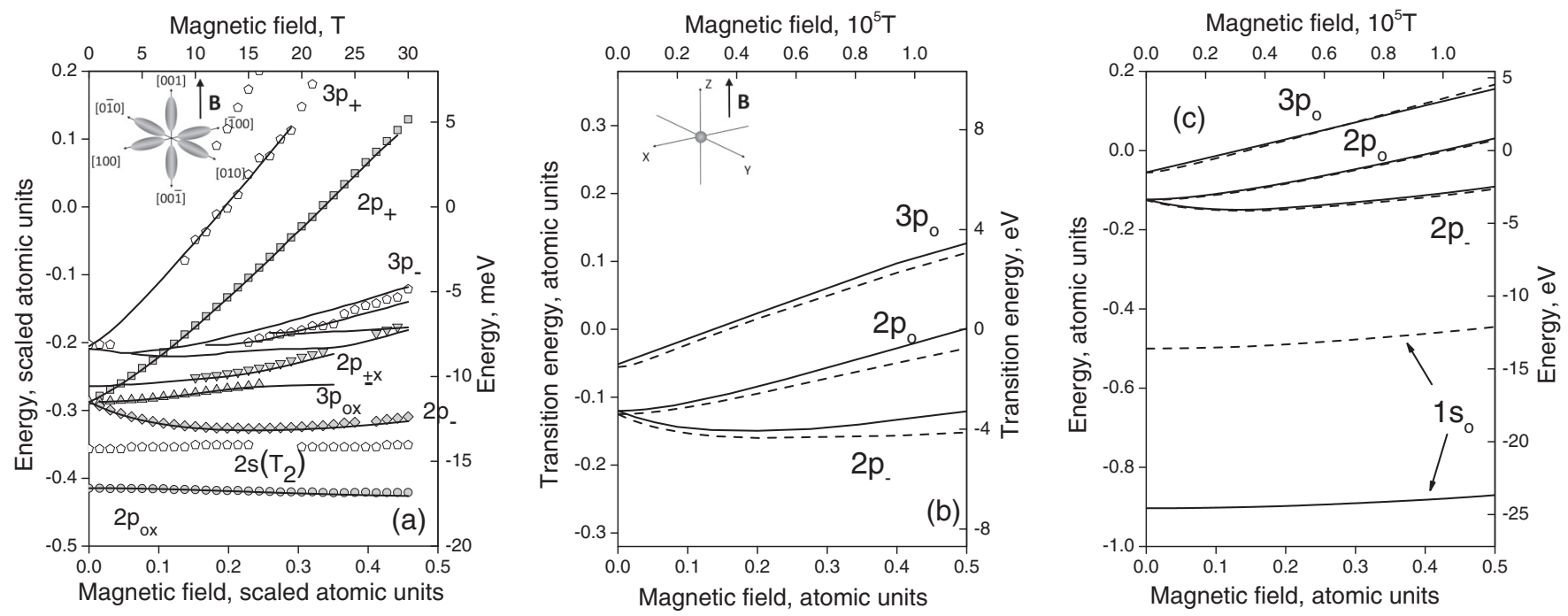

FIG. 2. The magnetic field dependence. (a) The measured Lyman series absorption lines in Si:Se (points) and Si:P (lines), in which for ease of comparison each transition energy has been rigidly shifted by the zero-field continuum edge energy (Se: $306.7 \mathrm{meV}$, P: $44.59 \mathrm{meV}$ ). Labels indicate the excited states for P Lyman series transitions from the $1 s\left(A_{1}\right)$ ground state. (b) Theoretical transition energy from the ground state to the excited states indicated on the figure for $\mathrm{H}$ (dashed line, e.g., $1 s_{0} \rightarrow 2 p_{0}$ ) and $\mathrm{He}$ (solid line, e.g., $1^{1} 0^{+} \rightarrow 1^{1} 0^{-}$) measured relative to the zero-field continuum edge energy (H: 0.5 a.u.; He: 0.9 a.u.). (c) Theoretical state energy for $\mathrm{H}$ (dashed line) and He (solid line) relative to the zero-field continuum edge. In the case of $\mathrm{H}$ we took the spin down binding energy from Ref. [10] and added $0.5 B$ to remove the effect of spin, and in the case of He we took the binding energy from Refs. [17] and [18] and subtracted it from the zero-field first ionization threshold followed by an additional $0.5 \mathrm{~B}$ for the continuum shift.

reachable by many high magnetic field laboratories. However, the large central cell correction for Si:Se greatly increases the binding energy, pushing the phase change to much higher magnetic fields of several hundred tesla, which is much more difficult to achieve.

\section{B. The ground states}

Having established that the odd-parity excited states are all the same in $\mathrm{P}, \mathrm{Se}$, and $\mathrm{Se}_{2}$, we now turn to the ground states, which may be examined with the difference between individual selenium and phosphorus transition lines. N.B.: Whereas Fig. 3(a) above shows the difference between $\mathrm{Se}$ and $\mathrm{P}$ transition splittings, we now investigate the difference between Se and P transitions, shown in Fig. 4(a). The figure shows that all of the lines behave in the same way, again confirming that the excited states of Se and $\mathrm{P}$ are identical. Therefore the shift observed in Fig. 4(a) is purely determined by the different field tuning of the selenium and phosphorus
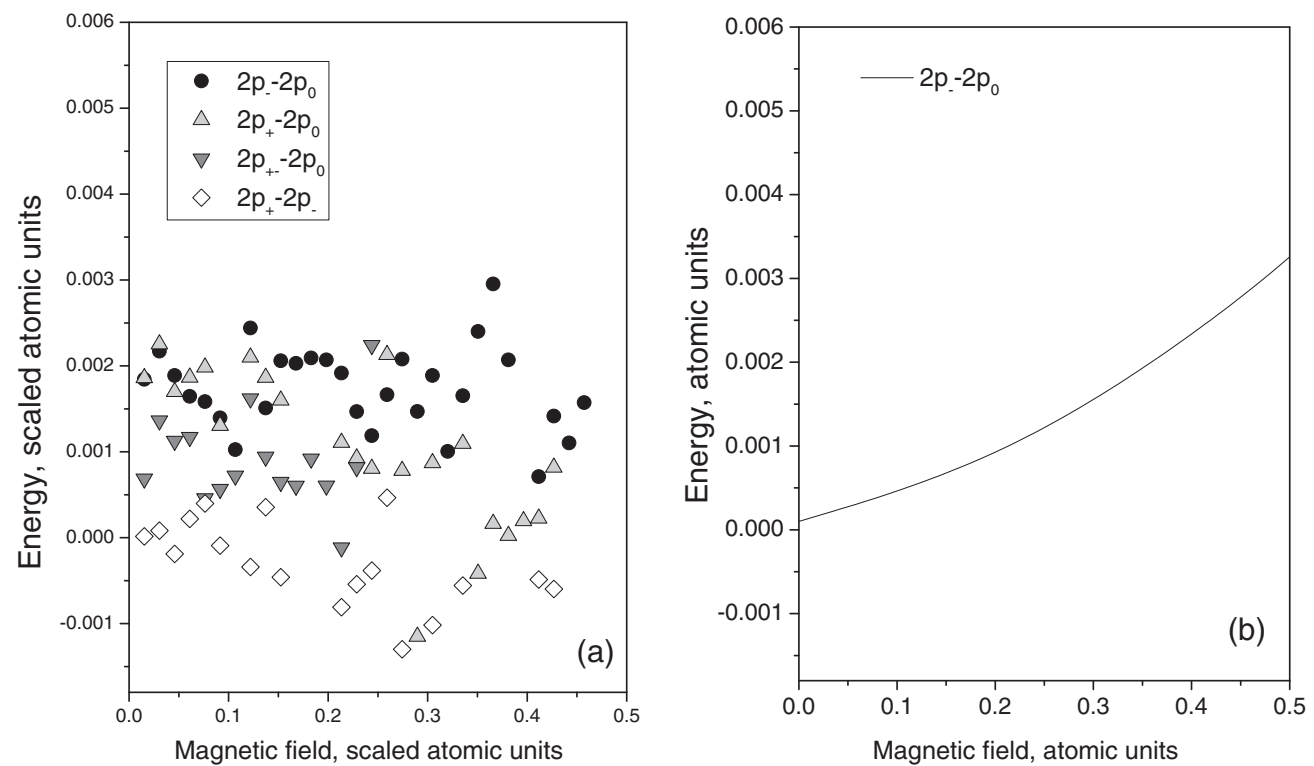

FIG. 3. Comparison between various transition splittings. (a) Se and $\mathrm{P}$, for example, $2 \mathrm{p}_{-}-2 p_{0}$ (black) shows the splitting between the $1 s-2 p_{-}$and $1 s-2 p_{0}$ transitions for Se minus the corresponding splitting for P. (b) He and H likewise. 

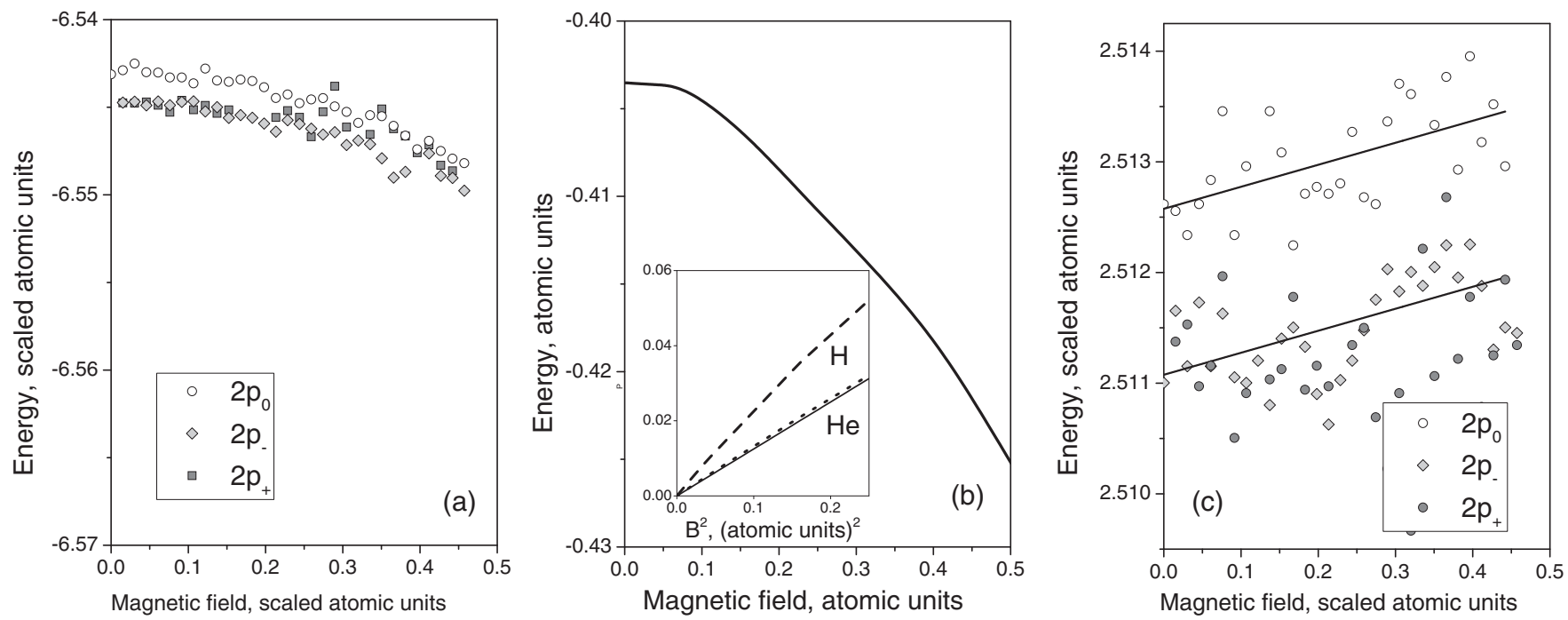

FIG. 4. Comparison between various transitions. (a) Se and P, for example, $2 p_{0}$ (circles) shows the $1 s-2 p_{0}$ transition for P minus the corresponding transition for Se. Assuming the excited states are identical, this is the same as the ground-state energy of Se minus that of P. (b) The ground-state energy of He minus that of $\mathrm{H}$. The inset of (b) shows the two ground states individually vs $B^{2}$, (dashed line) $\mathrm{H}$ and (solid line) $\mathrm{He}$, along with a line indicating $B^{2} / 8$ for reference (dotted line). (c) as (a) for $\mathrm{Se}_{1}$ and $\mathrm{Se}_{2}$. The solid lines are guides to the eye.

ground states. A similar effect is seen for the comparison between $\mathrm{Se}$ and $\mathrm{Se}_{2}$ [see Fig. 4(c)].

The difference in the ground-state energies calculated for hydrogen and helium is shown in Fig. 4(b) and demonstrates a monotonic drop. The effect only partly results from the differences in the quadratic Zeeman energy (QZE) due to the difference in size of the wave functions. The QZE is approximately $B^{2} r^{2} / 8$ from Eq. (1). Taking $\left\langle r^{2}\right\rangle \sim a_{0}^{2}$ for hydrogen (i.e., neglecting the change of size with $B$ ) gives a reasonable approximation. [On the Fig. 4(b) inset the dotted line for the approximation is close to the dashed line for the theory for H.] The QZE for helium is expected to be $\sim 30 \%$ of that for hydrogen due to the smaller size of the atom $(0.031 \mathrm{~nm}$ at zero field), while the field tuning is clearly larger due to the electron-electron interactions in He.

The $\mathrm{P}, \mathrm{Se}$, and $\mathrm{Se}_{2}$ centers have binding energies of 1.1 , 7.7 , and 5.2 scaled atomic units, respectively. In the case of $\mathrm{P}$, this gives an indication that the orbit radius is half that for scaled hydrogen and suggests that the $\mathrm{P}$ ground state has a QZE of about a quarter that found for scaled hydrogen [and indeed, Fig. 4(b) shows a field tuning about four times more than Fig. 4(a)], while the QZE for $\mathrm{Se}$ and $\mathrm{Se}_{2}$ are essentially zero because of the very small radius. The radius of the ground states of $\mathrm{Se}$ and $\mathrm{Se}_{2}$ are both less than the silicon bond length [13], and so the effective mass and dielectric medium approximations do not apply, meaning that there is no scaling of $B_{0}$ (and consequently the QZE is of order $\sim 10^{-7}$ of that for P). The field tuning observed in Fig. 4(c) is therefore entirely due to differences in the electron-electron interactions between $\mathrm{Se}$ and $\mathrm{Se}_{2}$. There is no reason for these interactions to be the same for both centers (one defect has two electrons and the other has four, and in each case these electrons are all within a very small interaction volume), and we infer that the differences in the interaction energies have the same order of magnitude as the absolute magnitudes. Given that the size of these interactions is of order $0.001 E_{h}$ at $0.5 B_{0}$, i.e., small compared with the field tunings indicated in Fig. 4(a), it seems likely that the latter are entirely due to the QZE for P.

That the effect of the electro-electron interaction is smallof the order of $0.001 E_{h}(40 \mu \mathrm{eV})$ at $0.5 B_{0}$ at $(30 \mathrm{~T})$ according to Fig. 4(c) - is not surprising, as it has been theoretically predicted for the helium molecule that this change become visible only at the fields higher than $5 B_{0}$ [7]. It should be mentioned that in comparison to the helium molecule, the electron-electron interaction in the $\mathrm{Se}_{2}$ center is expected to be magnified due to the fact that the separation (and consequently, the overlap of the electron wave functions) between the selenium atoms in silicon is 1 order of magnitude smaller in units of the electron ground-state orbital radius than in the case of the helium.

We note that exchange effects have been observed previously in the stress dependence of the $1 s\left(A_{1}\right) 1 s\left(A_{1}\right)$ to $1 s\left(A_{1}\right) 1 s\left(T_{2}\right)$ transition [9]. There is no atomic physics analog of these excited states which arise from the multivalley degeneracy of silicon. These states are locally $p$-like but have much smaller radius and hence more significant overlap with the core. With a finer field dependence it might be possible to observe the corresponding anticrossings, and this will be the subject of a future study.

The fact that these energy scales are very small may mean that $\mathrm{Se}$ is potentially useful as a control atom in the Stoneham-Fisher-Greenland quantum information scheme [5]. In this scheme the qubits are single donors (e.g., Bi) which are spatially separated and noninteracting. A control atom (such as the Se discussed here) is situated between the two qubits and excited optically. Exchange interaction between the qubits is much stronger when mediated by the excited control atom due to the increased wave-function overlap. If exchange and correlation internal to the Se were strong, the time evolution would be much more complex. The fact that the excited states are very closely hydrogenic also means that field tuning can be used to control the wave-function shape (and overlaps with 
neighbors) in the same way [1], with the additional benefit that midinfrared (rather than the far-infrared) lasers can be used for the optical excitation.

\section{C. $\mathrm{Se}_{2}$ as analog of $\mathrm{He}_{2}$}

It has to be said that $\mathrm{Se}_{2}$ can be considered only loosely an analog of $\mathrm{He}_{2}$. The helium molecule reduces its interatomic separation and becomes more strongly bound in magnetic fields, while the selenium atoms of the $\mathrm{Se}_{2}$ center occupy neighboring positions in the silicon lattice and do not move with magnetic field. There are two ways the adjacent selenium atoms influence each other. The main effect is that each atom introduces a strong local strain on the other [13], and this is not expected to depend on the magnetic field. Second, the electron-electron correlation and exchange interaction between the two pairs of electrons also modifies the binding energy, and as the electron wave function becomes more localized in the magnetic field, a decoupling of the Se atoms results, which adds additional field tuning on top of the other effects discussed above.

\section{CONCLUSION}

The selenium atom in silicon is in some ways an analog of the free helium atom. The energy and radius of the one-electron excited states in Se centers may be obtained from a hydrogenic model with scaled effective mass and dielectric constant. These states include an unexcited electron, which screens the ion so that the excited electron sees a singly positively charged core. The large distance between the two electrons renders the exchange and correlation negligible. We show that the odd-parity excited states of Si:Se behave identically to those of $\mathrm{Si}: \mathrm{P}$ at fields up to $30 \mathrm{~T}$. The ground states of selenium and phosphorus are quite different, and this is because of a combination of the quadratic Zeeman effect (which affects both but is stronger in $\mathrm{P}$ due to the smaller binding energy and larger extent of wave function) and the electron-electron correlation (which only affects selenium). A comparison between $\mathrm{Se}$ and $\mathrm{Se}_{2}$ centers allowed us to estimate the size of the electron-electron interaction and shows it to be of the order of $40 \mu \mathrm{eV}$ at $30 \mathrm{~T}\left(0.001 E_{h}\right.$ at $\left.0.5 B_{0}\right)$.

\section{ACKNOWLEDGMENTS}

We are grateful for conversations with I. Galbraith and P. McDonald (Heriot-Watt), as well as A. Fisher and P.T. Greenland (UCL). We gratefully acknowledge the support of the Engineering and Physical Sciences Research Council (EP/H026622/1 "COMPASSS"). B.N.M. is grateful to the Royal Society (for a Wolfson Research Merit Award). Part of this work has been supported by the Stichting voor Fundamenteel Onderzoek der Materie with financial support from the Nederlandse Organisatie voor Wetenschappelijk Onderzoek and from the European Commission/Cordis (228043 "EuroMagNET II").
[1] B. N. Murdin, Juerong Li, M. L. Y. Pang, E. T. Bowyer, K. L. Litvinenko, S. K. Clowes, H. Engelkamp, C. R. Pidgeon, I. Galbraith, N. V. Abrosimov, H. Riemann, S. G. Pavlov, H.-W. Hübers, and P. G. Murdin, Nat. Commun. 4, 1469 (2013).

[2] R. A. Lewis, A. Bruno-Alfonso, G. V. B. de Souza, R. E. M. Vickers, J. A. Colla, and E. Constable, Sci. Rep. 3, 3488 (2013).

[3] D. N. Batchelder and R. O. Simmons, J. Chem. Phys. 41, 2324 (1964); A. P. Zhernov, Low Temp. Phys. 26, 908 (2000).

[4] K. Saeedi, S. Simmons, J. Z. Salvail, P. Dluhy, H. Riemann, N. V. Abrosimov, P. Becker, H.-J. Pohl, J. J. L. Morton, and M. L. W. Thewalt, Science 342, 830 (2013).

[5] A. M. Stoneham, A. J. Fisher, and P. T. Greenland, J. Phys.: Condens. Matter 15, L447 (2003).

[6] P. Schmelcher and W. Schweizer, Atoms and Molecules in Strong External Fields, eBook edn. (Kluwer Academic Publishers, Dordrecht, 2002).

[7] P. Schmelcher, M. V. Ivanov, and W. Becken, Phys. Rev. A 59, 3424 (1999).
[8] K. K. Lange, E. I. Tellgren, M. R. Hoffmann, and T. Helgaker, Science 337, 327 (2012).

[9] G. Grossman, K. Bergman, and M. Kleverman, Physica B 146, 30 (1987)

[10] W. Becken, P. Schmelcher, and F. K. Diakonos, J. Phys. B: At. Mol. Opt. Phys. 32, 1557 (1999)

[11] Yu. A. Astrov, V. B. Shuman, L. M. Portsel, and A. N. Lodygin, Semiconductors 48, 413 (2014).

[12] E. Janzen, R. Stedman, G. Grossmann, and H. G. Grimmeiss, Phys. Rev. B 29, 1907 (1984); M. Steger, A. Yang, and M. L. W. Thewalt et al., ibid. 80, 115204 (2009).

[13] Y. Mo, M. Z. Bazant, and E. Kaxiras, Phys. Rev. B 70, 205210 (2004).

[14] H. G. Grimmeiss, E. Janzen, and K. Larsson, Phys. Rev. B 25, 2627 (1982).

[15] W. Rosner, G. Wunner, H. Herold, and H. Ruder, J. Phys. B: At. Mol. Phys. 17, 29 (1984).

[16] A. Scrinzi, Phys. Rev. A 58, 3879 (1998).

[17] W. Becken and P. Schmelcher, J. Phys. B: At. Mol. Opt. Phys. 33, 545 (2000).

[18] W. Becken and P. Schmelcher, Phys. Rev. A 63, 053412 (2001). 